\title{
Utility of the Lactate/Albumin Ratio as a Predictor for Mortality in Necrotizing Fasciitis Patients
}

\author{
Kiew-Kii Lau, ${ }^{1}$ Cheng-Ting Hsiao, ${ }^{1,2}$ Wen-Chih Fann, ${ }^{1}$ and Chia-Peng Chang ${ }^{1}{ }^{1}$ \\ ${ }^{1}$ Department of Emergency Medicine, Chang Gung Memorial Hospital, Chiayi, Taiwan \\ ${ }^{2}$ Department of Medicine, Chang Gung University, Taoyuan, Taiwan \\ Correspondence should be addressed to Chia-Peng Chang; giovanni850730@gmail.com
}

Received 19 July 2021; Accepted 7 October 2021; Published 13 October 2021

Academic Editor: Yan-Ren Lin

Copyright ( $) 2021$ Kiew-Kii Lau et al. This is an open access article distributed under the Creative Commons Attribution License, which permits unrestricted use, distribution, and reproduction in any medium, provided the original work is properly cited.

Background. The lactate/albumin (L/A) ratio has been proposed as a prognostic marker because the ratio is associated with multiple organ failure and mortality in critically ill patients. We aimed to investigate the clinical utility of the L/A ratio as a good prognostic indicator of mortality in a cohort of necrotizing fasciitis patients. Method. This retrospective study was conducted in two tertiary hospitals in Taiwan between 2015 and 2020. We reviewed adult patients with measured serum lactate and albumin on the emergency department (ED) arrival to evaluate the prognostic performance of the lactate and lactate/albumin (L/A) ratio for outcome prediction. Result. Of the 262 NF patients, $20(7.63 \%)$ died in the hospital. The area under the receiver operating characteristic curve (AUROC) value of the L/A ratio $(0.76,95 \%$ confidence interval $[\mathrm{CI}] 0.69-0.81, P<0.01)$ was higher than lactate alone $(0.71,95 \%$ CI $0.65-0.74 P<0.01)$ for predicting in-hospital mortality. The optimal cutoff of the L/A ratio was 1.61 . The AUROC value of the L/A ratio was better than lactate alone regardless of normal lactate level. The cutoff of L/A ratio and hypoalbuminemia showed further discriminative value for in-hospital mortality even in patients with normal lactate levels. Conclusion. The prognostic performance of the L/A ratio was superior to a single measurement of lactate for predicting in-hospital mortality and intensive care unit (ICU) lengths in necrotizing fasciitis patients. Aggressive intervention and intensive care were necessary for high-risk NF patients upon ED arrival.

\section{Introduction}

Necrotizing fasciitis (NF) is a rare, rapidly progressive infection of subcutaneous tissue and fascia, characterized by widespread fascial necrosis [1]. The treatment of choice for $\mathrm{NF}$ is rapid surgical debridement and broad-spectrum antibiotic therapy [2]. Even under rapid and timely management, the risk of mortality and morbidity, such as amputation and multiorgan dysfunction, remains high [3-5]. Therefore, predictive biomarkers of mortality in NF patients are important for timely management.

Among widely used laboratory markers, band polymorphonuclear neutrophils $>10 \%$, serum creatinine level $>2 \mathrm{mg} / \mathrm{dL}$, hyperlactatemia, Laboratory Risk Indicator of Necrotizing Fasciitis (LRINEC) score $>8$, and serum albumin levels have been identified in association with mortality [6-8]. Elevated lactate was shown to be independently associated with mortality rate in critical ill patients [9]. Even intermediate initial serum lactate was an indicator of mortality of organ dysfunction and shock in severe sepsis patients in the emergency department (ED) [10]. In hospitalized patients, increased lactate indicated high mortality, mechanical ventilation, vasopressor requirement, and a high incidence of ICU admission [11-13]. Besides, serum albumin levels may also serve as a prognostic factor for critical patients, and the diagnostic performance of this parameter in critical care is well known [14-16]. The clinical significance of serum lactate and albumin was established over the last decade. Trends in lactate concentration over time reflect the clinical response of patients to resuscitation and surgical intervention. However, lactate and albumin level is not routinely monitored at the time of patient arrival as standard of care. Emergency department (ED) physicians are concerned with the resuscitation and identification risk of NF 
patients. Early recognition of NF patients who are at high risk for mortality allows for timely changes in therapy and improves the overall outcomes. We try to measure the association of the initial serum lactate and albumin level in the ED with in-hospital mortality of NF. Our objective in this study was to retrospectively evaluate whether the lactate/ albumin ratio in ED is associated with increased in-hospital mortality in NF patients. The lactate/albumin (L/A) ratio has been proposed as a prognostic marker because the ratio is associated with multiple organ failure and mortality in critically patients $[17,18]$. However, the prognostic performance of L/A ratio has not yet been reported among patients with necrotizing fasciitis. In this study, we evaluated the prognostic performance of the L/A ratio and lactate levels for predicting outcome in NF.

\section{Materials and Methods}

2.1. Patient Selection. The institutional review board of two hospitals approved this study. We followed the method of Shin et al.'s study [19]. The medical records of patients who met the inclusion criteria of surgically proven NF and who received management between 2015 and 2020 in two tertiary hospitals were reviewed. Selected comorbidities and initial laboratory values were extracted through medical chart review. All patients were assessed by emergency physicians as soon as they were admitted. They received broad-spectrum antibiotic treatment for anaerobic and aerobic bacteria as well as early surgical debridement including fasciotomy or primary amputation after diagnosis. Each patient's medical record was screened for documentation of NF to confirm the diagnosis. Blood samples were collected in the ED upon arrival, so the lactate and albumin levels were determined. Variables were compared between survivors and nonsurvivors groups.

2.2. Data Collection and Measurement. Age, sex, vital signs in the ED, the presence of comorbidities (i.e., heart disease, pulmonary disease, liver disease, kidney disease, and diabetes), serum lactate, albumin, and SOFA score were analyzed. After data collection was completed, random chart reviews were performed to ensure accuracy. Patients with inhospital mortality were defined as a death occurring in the hospital after admission, also known as the 'nonsurvivors group', and otherwise as the 'survivors group.' We defined these variables as follows: shock status, episodes of systolic blood pressure less than $90 \mathrm{mmHg}$ at the ED; hypothermia, body temperature less than $36^{\circ} \mathrm{C}$ at the $\mathrm{ED}$; hyperthermia, body temperature $\geq 38^{\circ} \mathrm{C}$ at the $\mathrm{ED}$; acidosis, $\mathrm{pH}$ less than 7.35 in the arterial blood gas test at the ED; coagulopathy, a prolonged prothrombin time test (international normalized ratio) result greater than 1.5 or platelet counts less than $100 \times 10^{3} \mathrm{uL}$ at the ED; and hypoxia, episodes of $\mathrm{SpO} 2<90 \%$, oxygen saturation less than $90 \%$ at the ED. Prediction accuracy of in-hospital mortality was assessed using the area under the receiver operating characteristic (AUROC) curve. Prognostic accuracy was assessed in all patients and in subgroups based on lactate levels. For comparison of AUROC curves of two diagnostic modalities (L/A ratio, lactate), we used a test for dependent receiver operating characteristic curves. The cutoff point was determined using the Liu method that maximizes sensitivity and specificity. To adjust for potential confounders in the association between the L/A ratio and in-hospital mortality, individual factors with a $P$ value less than 0.05 were used for multiple logistic regression. Statistical significance was defined by a $P$ value $<0.05$.

\section{Results}

Baseline characteristics of survivors and nonsurvivors groups are listed in Table 1. The overall in-hospital mortality was $7.63 \%$ in all the patients. The overall median age of patients was 59.8 (IQR 50.2-75.4), and 163 (62.2\%) patients were male. The overall median maximum SOFA score initially was 7 (IQR 5-11). In laboratory tests, the nonsurvivor group showed lower albumin levels (median, $2.6 \mathrm{~g} /$ $\mathrm{dL}$ vs $3.1 \mathrm{~g} / \mathrm{dL}$ ), higher lactate levels (median, $4.9 \mathrm{mmol} / \mathrm{L}$ vs $2.8 \mathrm{mmol} / \mathrm{L}$ ), and higher L/A ratio (median, $1.8 \mathrm{vs} 1.1$ ) than the survival group $(P<0.05$, all). Table 2 shows comparisons between AUROC values and the cutoff point of the L/A ratio for in-hospital mortality. The AUROC value of the L/A ratio for predicting mortality $(0.76,95 \%$ confidence interval $(\mathrm{CI})$ $0.70-0.82 ; P<0.01)$ was higher than that for lactate alone (0.71, 95\% CI 0.65-0.77; $P<0.01)$. The AUROC value of the $\mathrm{L} / \mathrm{A}$ ratio was significantly higher than that of lactate regardless of lactate level (normal: 0.75 , 95\% CI $0.70-0.80$ vs. 0.64, 95\% CI 0.59-0.69, $P<0.01$; intermediate: $0.69,95 \%$ CI $0.63-0.75$ vs. $0.59,95 \%$ CI $0.53-0.75, P<0.01$; and high: $0.72,95 \%$ CI $0.68-0.76$ vs. 0.68 , 95\% CI $0.64-0.72$, $P=0.015)$. Table 3 compared in-hospital mortality according to initial lactate level using the cutoff point for L/A ratio and albumin level. The mortality rates were significantly higher when the L/A ratio was above the cutoff point regardless of lactate level (normal: $6.9 \%$ vs. $2.2 \%, P<0.01$; intermediate: $13.0 \%$ vs. $3.9 \%, P<0.01$; and high: $15.6 \%$ vs. $7.2 \%, P<0.01)$. A significant trend in higher mortality was also observed in hypoalbuminemia patients compared with patients with normal albumin. After adjusting for several confounding factors such as shock, acidosis, SOFA score, chronic kidney disease, and diabetes, we found a significant association between the L/A ratio and in-hospital mortality (adjusted odds ratio (OR) 1.48, 95\% CI 1.30-1.75; $P<0.01$; Table 4).

\section{Discussion}

The diagnostic performance of the L/A ratio was better than that of single lactate for predicting in-hospital mortality in NF patients. Improved prognostic accuracy was consistent in subgroups of lactate levels (low, intermediate, and high), the AUROC of the L/A ratio was relatively fair for predicting mortality (AUROC $\geq 0.70$ ). In addition, the $\mathrm{L} / \mathrm{A}$ ratio cutoff and hypoalbuminemia showed further discriminative value for in-hospital mortality than single lactate even in patients with normal or intermediate lactate levels. Many studies have demonstrated that either admission lactate or peak 
TABLE 1: Comparison of baseline characteristics between survival and nonsurvival groups.

\begin{tabular}{|c|c|c|c|}
\hline Characteristics & Survivors $(n=242)$ & Nonsurvivors $(n=20)$ & $P$ value \\
\hline Age (years) & $58.3(39.1-62.3)$ & $60.7(41.4-79.5)$ & 0.89 \\
\hline Sex (male) & $150(62.0 \%)$ & $13(65.0 \%)$ & 0.57 \\
\hline \multicolumn{4}{|l|}{ ED vital signs } \\
\hline Hypoxia & $24(10.0 \%)$ & $3(15.0 \%)$ & 0.07 \\
\hline Shock & $49(20.2 \%)$ & $6(30.0 \%)$ & $<0.01$ \\
\hline Hyperthermia & $143(59.1 \%)$ & $12(60.0 \%)$ & 0.91 \\
\hline Hypothermia & $43(17.8 \%)$ & $3(15.0 \%)$ & 0.84 \\
\hline SOFA score & $4(0-6)$ & $9(5-23)$ & $<0.01$ \\
\hline \multicolumn{4}{|l|}{ Laboratory test } \\
\hline Acidosis & $45(18.6 \%)$ & $6(30.0 \%)$ & 0.02 \\
\hline Coagulopathy & $39(16.1 \%)$ & $4(20.0 \%)$ & 0.44 \\
\hline Lactate $(\mathrm{mmol} / \mathrm{l})$ & $2.8(0.5-5.6)$ & $4.9(1.2-9.8)$ & $<0.01$ \\
\hline Albumin $(\mathrm{g} / \mathrm{dl})$ & $3.1(2.1-4.8)$ & $2.6(1.9-3.6)$ & $<0.01$ \\
\hline Lactate/albumin ratio & $1.1(0.8-1.8)$ & $1.8(1.1-2.8)$ & $<0.01$ \\
\hline \multicolumn{4}{|l|}{ Comorbidities } \\
\hline Heart failure & $38(15.7 \%)$ & $3(15.0 \%)$ & 0.97 \\
\hline Diabetes mellitus & $63(26.0 \%)$ & $7(35.0 \%)$ & 0.04 \\
\hline Liver cirrhosis & $48(19.8 \%)$ & $4(20.0 \%)$ & 0.15 \\
\hline Chronic kidney disease & $75(31.0 \%)$ & $9(45.0 \%)$ & 0.01 \\
\hline Chronic lung disease & $29(12.0 \%)$ & $2(10.0 \%)$ & 0.66 \\
\hline ICU lengths (day) & $5.4(2.1-11.8)$ & $9.2(1.5-19.7)$ & $<0.01$ \\
\hline
\end{tabular}

Values are represented as median (interquartile range). Numbers in parentheses denote percentages. ED, emergency department; ICU, intensive care unit; SOFA, sequential organ failure assessment.

TABLE 2: AUROC and L/A ratio cutoff value for in-hospital mortality.

\begin{tabular}{|c|c|c|c|c|c|c|}
\hline & \multicolumn{3}{|c|}{$\begin{array}{l}\text { AUROC for in-hospital mortality (95\% } \\
\text { confidence interval) }\end{array}$} & \multicolumn{3}{|c|}{ Lactate/albumin ratio cutoff point } \\
\hline & Lactate & L/A ratio & $P$ & Cutoff value & Sensitivity & Specificity \\
\hline All patients & $0.71(0.65-0.77)$ & $0.76(0.70-0.82)$ & $<0.01$ & 1.61 & 0.75 & 0.81 \\
\hline Lactate level (mmol/L) & & & & & & \\
\hline Normal lactate $(<2.0)$ & $0.64(0.59-0.69)$ & $0.75(0.70-0.80)$ & $<0.01$ & 0.75 & 0.76 & 0.82 \\
\hline Intermediate lactate $(2.0 \leqq x<4.0)$ & $0.59(0.53-0.75)$ & $0.69(0.63-0.75)$ & $<0.01$ & 1.36 & 0.63 & 0.85 \\
\hline High lactate $(\geqq 4.0)$ & $0.68(0.64-0.72)$ & $0.72(0.68-0.76)$ & 0.015 & 2.48 & 0.71 & 0.83 \\
\hline
\end{tabular}

lactate concentration is associated with mortality in adults. Our results are in line with recently published findings that suggest that blood lactate concentration upon ED arrival is predictive of mortality, independent of SOFA score $[10,20-22]$. Some theories exist regarding the reasons why lower serum albumin levels, or hypoalbuminemia, may be associated with poor outcomes. Because synthesis and distribution of albumin may be directly associated with serum albumin level, factors that could affect albumin synthesis, distribution, or both need to be considered. The $\mathrm{L} / \mathrm{A}$ ratio has been proposed as a marker for combined interpretation of lactate and albumin levels; some studies have investigated its usefulness as a prognostic marker in sepsis or ICU patients $[23,24]$. To the best of our knowledge, our study is the first to evaluate the L/A ratio and in-hospital mortality for NF patients.

The L/A ratio has potential benefits for prognostication of NF patients considering the results from our study and previous studies. The two markers independently predict mortality, and a combination of both parameters might increase the predictive value. Furthermore, normal or intermediate levels of lactate might be misinterpreted as a good prognosis. The L/A ratio might be used for additional identification of highrisk patients. In addition, our study suggests that a simple approach might provide further prognostic information using hypoalbuminemia and categories of lactate level.

There were several limitations of this study. First, this study was retrospective in nature and, thus, has inherent limitations with regards to selection bias. The authors who were aware of the potential biases held multiple meetings to ensure patients were correctly identified. Second, the data were collected from two centers limiting the generalizability of these results. The validity of the result still requires other external validation to confirm. Third, we only included patients on whom lactate and albumin were both taken. Finally, we did not have enough information to compare the performance of the L/A ratio with other scoring systems such as APACHE II. 
TABLE 3: In-hospital mortality according to lactate level, lactate/ albumin (L/A) ratio, and albumin level.

\begin{tabular}{|c|c|c|}
\hline & $\begin{array}{l}\text { In-hospital } \\
\text { mortality }\end{array}$ & $P$ \\
\hline \multicolumn{3}{|l|}{ Lactate and $\mathrm{L} / \mathrm{A}$ ratio } \\
\hline Normal lactate level & & $<0.01$ \\
\hline $\begin{array}{l}\text { L/A ratio }<\text { cutoff point }(0.75) \\
(n=45)\end{array}$ & $1(2.2 \%)$ & \\
\hline $\begin{array}{l}\mathrm{L} / \mathrm{A} \text { ratio } \geqq \text { cutoff point }(0.75) \\
(n=29)\end{array}$ & $2(6.9 \%)$ & \\
\hline & $<0.01$ \\
\hline $\begin{array}{l}\text { L/A ratio < cutoff point }(1.36) \\
(n=51)\end{array}$ & $2(3.9 \%)$ & \\
\hline $\begin{array}{l}\mathrm{L} / \mathrm{A} \text { ratio } \geqq \text { cutoff point }(1.36) \\
(n=23)\end{array}$ & $3(13.0 \%)$ & \\
\hline & $<0.01$ \\
\hline $\begin{array}{l}\text { L/A ratio }<\text { cutoff point }(2.48) \\
(n=69)\end{array}$ & $5(7.2 \%)$ & \\
\hline $\begin{array}{l}\mathrm{L} / \mathrm{A} \text { ratio } \geqq \text { cutoff point }(2.48) \\
(n=45)\end{array}$ & $7(15.6 \%)$ & \\
\hline \multicolumn{3}{|l|}{ Lactate and albumin level } \\
\hline Normal lactate level & & $<0.01$ \\
\hline Albumin $<3.0(n=33)$ & $2(6.1 \%)$ & \\
\hline Albumin $\geqq 3.0 \quad(n=41)$ & $1(2.4 \%)$ & \\
\hline \multicolumn{2}{|l|}{ Intermediate lactate level } & $<0.01$ \\
\hline Albumin $<3.0(n=39)$ & $4(10.3 \%)$ & \\
\hline Albumin $\geqq 3.0(n=35)$ & $1(2.9 \%)$ & \\
\hline \multicolumn{2}{|l|}{ High lactate level } & $<0.01$ \\
\hline Albumin $<3.0(n=60)$ & $8(13.3 \%)$ & \\
\hline Albumin $\geqq 3.0(n=54)$ & $4(7.4 \%)$ & \\
\hline
\end{tabular}

TABLE 4: Multivariable logistic regression.

\begin{tabular}{lccc}
\hline & Odds ratio & $95 \%$ CI & $P$ \\
\hline Shock & 1.02 & $1.00-1.04$ & 0.19 \\
Acidosis & 0.98 & $0.82-1.14$ & 0.58 \\
Chronic kidney disease & 1.35 & $1.29-1.41$ & 0.02 \\
Diabetes mellitus & 1.74 & $1.25-2.16$ & 0.26 \\
SOFA score & 1.21 & $1.14-1.19$ & 0.01 \\
Lactate/albumin ratio & 1.48 & $1.30-1.75$ & $<0.01$ \\
\hline
\end{tabular}

\section{Conclusions}

The prognostic performance of the L/A ratio was better than a single lactate measurement for predicting in-hospital mortality in NF patients. This ratio was a useful prognostic factor regardless of initial lactate level. Aggressive intervention and intensive care were necessary for high-risk NF patients upon ED arrival.

\section{Abbreviations}

AUROC:: Area under the receiver operating characteristic curve

CI: $\quad$ Confidence interval

ED: $\quad$ Emergency department

NF: $\quad$ Necrotizing fasciitis

OR: $\quad$ Odds ratio

SOFA: Sequential organ failure assessment.

\section{Data Availability}

Data are available on request.

\section{Ethical Approval}

The institutional review board of Chia-yi Chang Gung Memorial Hospital approved this retrospective study, (1004178B) and (201900447B0C601).

\section{Conflicts of Interest}

The authors declare no conflicts of interest.

\section{Authors' Contributions}

KK Lau conceived the study, participated in its design and coordination, and helped to draft the manuscript. CT Hsiao participated in drafting the manuscript and statistical analysis. WC Fann participated in the design of the study and statistical analysis. CP Chang participated in supervision. All authors read and approved the final manuscript.

\section{Acknowledgments}

The study was supported by the Chang Gung Memorial Hospital research program with grant nos. CORPG6E0043, CMRPG6J0111, and CMRPG6J0112.

\section{References}

[1] C.-N. Lin, C.-T. Hsiao, C.-P. Chang et al., "The relationship between fluid accumulation in ultrasonography and the diagnosis and prognosis of patients with necrotizing fasciitis," Ultrasound in Medicine \& Biology, vol. 45, no. 7, pp. 15451550, 2019.

[2] C. R. McHenry, J. J. Piotrowski, D. Petrinic, and M. A. Malangoni, "Determinants of mortality for necrotizing soft-tissue infections," Annals of Surgery, vol. 221, no. 5, pp. 558-565, 1995.

[3] A. G. Angoules, G. Kontakis, E. Drakoulakis, G. Vrentzos, M. S. Granick, and P. V. Giannoudis, "Necrotising fasciitis of upper and lower limb: a systematic review," Injury, vol. 38, no. Suppl5, pp. S19-S26, 2007.

[4] D. A. Anaya, K. McMahon, A. B. Nathens, S. R. Sullivan, H. Foy, and E. Bulger, "Predictors of mortality and limb loss in necrotizing soft tissue infections," Archives of Surgery, vol. 140, no. 2, pp. 151-157, 2005.

[5] A. K. Khanna, S. K. Tiwary, P. Kumar, R. Khanna, and A. Khanna, "A case series describing 118 patients with lower limb necrotizing fasciitis," The International Journal of Lower Extremity Wounds, vol. 8, no. 2, pp. 112-116, 2009.

[6] K.-F. Huang, M.-H. Hung, Y.-S. Lin et al., "Independent predictors of mortality for necrotizing fasciitis: a retrospective analysis in a single institution," The Journal of Trauma, Injury, Infection, and Critical Care, vol. 71, no. 2, pp. 467-473, 2011.

[7] C.-T. Hsiao, H.-H. Weng, Y.-D. Yuan, C.-T. Chen, and I.-C. Chen, "Predictors of mortality in patients with necrotizing fasciitis," The American Journal of Emergency Medicine, vol. 26, no. 2, pp. 170-175, 2008.

[8] C.-P. Chang, C.-T. Hsiao, C.-N. Lin, and W.-C. Fann, "Risk factors for mortality in the late amputation of necrotizing 
fasciitis: a retrospective study," World Journal of Emergency Surgery, vol. 13, no. 1, p. 45, 2018.

[9] H. Khosravani, R. Shahpori, H. T. Stelfox, A. W. Kirkpatrick, and K. B. Laupland, "Occurrence and adverse effect on outcome of hyperlactatemia in the critically ill," Critical Care, vol. 13, no. 3, p. R90, 2009.

[10] M. E. Mikkelsen, A. N. Miltiades, D. F. Gaieski et al., "Serum lactate is associated with mortality in severe sepsis independent of organ failure and shock," Critical Care Medicine, vol. 37, no. 5, pp. 1670-1677, 2009.

[11] Y. Tang, J. Choi, D. Kim et al., "Clinical predictors of adverse outcome in severe sepsis patients with lactate 2 to $4 \mathrm{mM}$ admitted to the hospital," An International Journal of Medicine, vol. 108, no. 4, pp. 279-287, 2014.

[12] C.-P. Chang, W.-C. Fann, S.-R. Wu, C.-N. Lin, and C.-T. Hsiao, "Lactate on emergency department arrival as a predictor of in-hospital mortality in necrotizing fasciitis: a retrospective study," Journal of Orthopaedic Surgery and Research, vol. 14, no. 1, p. 73, 2019.

[13] J. Bakker, P. Gris, M. Coffernils, R. J. Kahn, and J.-L. Vincent, "Serial blood lactate levels can predict the development of multiple organ failure following septic shock," The American Journal of Surgery, vol. 171, no. 2, pp. 221-226, 1996.

[14] M. E. Jellinge, D. P. Henriksen, P. Hallas, and M. Brabrand, "Hypoalbuminemia is a strong predictor of 30-day all-cause mortality in acutely admitted medical patients: a prospective, observational, cohort study," PLoS One, vol. 9, no. 8, Article ID e105983, 201422.

[15] D. Barchel, D. Almoznino-Sarafian, M. Shteinshnaider, I. Tzur, N. Cohen, and O. Gorelik, "Clinical characteristics and prognostic significance of serum albumin changes in an internal medicine ward," European Journal of Internal Medicine, vol. 24, no. 8, pp. 772-778, 2013.

[16] C.-P. Chang, W.-C. Fann, S.-R. Wu, C.-N. Lin, I.-C. Chen, and a. C.-T. Hsiao, "Diagnostic performance of initial serum albumin level for predicting in-hospital mortality among necrotizing fasciitis patients," Journal of Clinical Medicine, vol. 7 , no. 11 , p. $435,2018$.

[17] M. Müllner, F. Sterz, H. Domanovits, W. Behringer, M. Binder, and A. N. Laggner, "The association between blood lactate concentration on admission, duration of cardiac arrest, and functional neurological recovery in patients resuscitated from ventricular fibrillation," Intensive Care Medicine, vol. 23, no. 11, pp. 1138-1143, 1997.

[18] W. E. Owen, J. E. Caron, and J. R. Genzen, "Liver function testing on the abaxis piccolo Xpress: use in Ebola virus disease protocols," Clinica Chimica Acta, vol. 446, pp. 119-127, 2015.

[19] J. Shin, S. Y. Hwang, I. J. Jo et al., "Prognostic value of the lactate/albumin ratio for predicting 28-day mortality in critically ILL sepsis patients," Shock, vol. 50, no. 5, pp. 545-550, 2018.

[20] O. Kruse, N. Grunnet, and C. Barfod, "Blood lactate as a predictor for in-hospital mortality in patients admitted acutely to hospital: a systematic review," Scandinavian Journal of Trauma, Resuscitation and Emergency Medicine, vol. 19, no. 1, p. 74, 2011.

[21] J. Martin, M. Blobner, R. Busch, N. Moser, E. Kochs, and P. B. Luppa, "Point-of-care testing on admission to the intensive care unit: lactate and glucose independently predict mortality," Clinical Chemistry and Laboratory Medicine, vol. 51, pp. 405-412, 2013.

[22] L. Gustavsson, L.-M. Andersson, M. Brink, M. Lindh, and J. Westin, "Venous lactate levels can be used to identify patients with poor outcome following community-onset norovirus enteritis," Scandinavian Journal of Infectious Diseases, vol. 44, no. 10, pp. 782-787, 2012.

[23] B. Wang, G. Chen, Y. Cao, J. Xue, J. Li, and Y. Wu, "Correlation of lactate/albumin ratio level to organ failure and mortality in severe sepsis and septic shock," Journal of Critical Care, vol. 30, no. 2, pp. 271-275, 2015.

[24] M. Lichtenauer, B. Wernly, B. Ohnewein et al., "A valuable tool for risk stratification in septic patients admitted to ICU," International Journal of Molecular Sciences, vol. 18, no. 9, 1893 pages, 2017. 